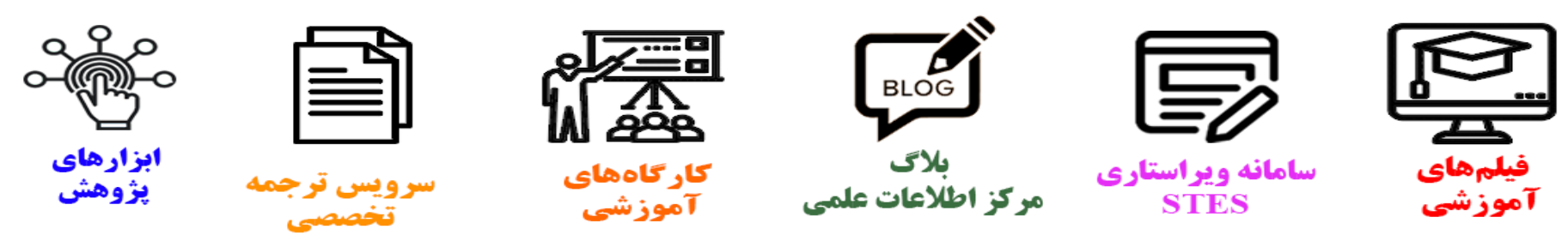

\title{
(c)
}

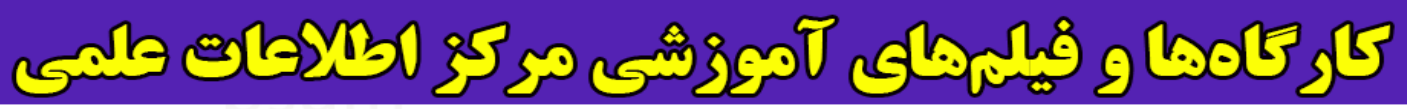
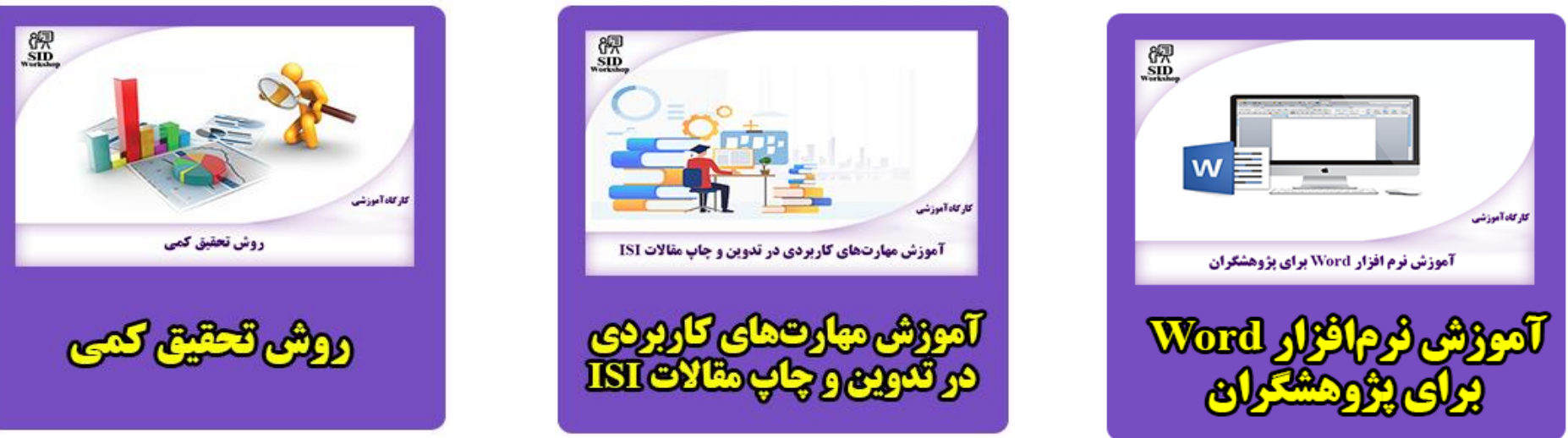


\title{
International Journal of Engineering
}

Journal Homepage: www.ije.ir

\section{Analytical Investigation of Jeffery-Hamel Flow with High Magnetic Field and Nano Particle by RVIM}

\author{
A. A. Imani a , Y. Rostamian b, D. D. Ganji c , H. B. Rokni*d \\ a Islamic Azad University, Babol Branch, Babol, Iran \\ ${ }^{b}$ Faculty of Mechanical Engineering, Islamic Azad University, Sari Branch, Sari, Iran \\ c Department of Mechanical Engineering, Babol University of Technology, P.O. Box 484, Babol, Iran. \\ ${ }^{d}$ University of Denver, Mechanical and Materials Engineering, 2390 S York St., Denver, CO, USA.
}

\section{$P A P E R \quad I N F O$}

Paper history:

Received 23 June 2012

Received in revised form 25 August 2012

Accepted in revised form 30 August 2012

\section{Keywords:}

Magneto Hydro Dynamic

Jeffery-hamel Flow

RVIM

Nonlinear Ordinary Differential Equation Nanofluid

\section{$A B S T R A C T$}

Many researchers have been interested in application of mathematical methods to find analytical solutions of nonlinear equations and for this purpose, new methods have been developed. One of the newest analytical methods to solve nonlinear equations is Reconstruction of variational Iteration Method (RVIM) which is an accurate and a rapid convergence method in finding the approximate solution for nonlinear equations. By applying Laplace Transform, RVIM overcomes the difficulty of the perturbation techniques and other variational methods in case of using small parameters and Lagrange multipliers, respectively. In this study RVIM is applied for the effects of magnetic field and nano particle on the Jeffery-hamel flow. The traditional Navier-Stokes equation of fluid mechanics and Maxwell's electromagnetism governing equations are reduced to nonlinear ordinary differential equations to model the problem. The flow field inside the divergent channel is studied for various values of Hartmann number and angle of channel. Finally the effect of nano particle volume fraction in the absence of magnetic field is investigated, too. The validity of RVIM method is ascertained by comparing our results with numerical (Runge Kutta method) results.

doi: 10.5829/idosi.ije.2012.25.03c.09

\begin{tabular}{|c|c|c|c|}
\hline$A^{*}$ & Constant parameter & \\
\hline$B_{0}$ & Magnetic field(wb. $\left.\mathrm{m}^{-2}\right)$ & $\alpha$ & Angle of the channel \\
\hline$F$ & General nonlinear operator & $\eta$ & Dimensionless angle \\
\hline$L u$ & Linear term & $\theta$ & Any angle \\
\hline$R u$ & The remained of linear operator & $\rho$ & Density \\
\hline$A$ & Adomian polynominal & $\phi$ & Nanoparticle volume fraction \\
\hline$f(\eta)$ & Dimensionless velocity & $\mu$ & Dynamic viscosity \\
\hline$H a$ & Hartmann number & $v$ & Kinematic viscosity \\
\hline Pressure term & Pressure term & $\beta$ & Constant \\
\hline$R e$ & Reynolds number & & \\
\hline$r, \theta$ & Cylindrical coordinates & \multicolumn{2}{|c|}{ Subscripts } \\
\hline$U_{\max }$ & Maximum value of velocity & $\infty$ & Condition at infinity \\
\hline \multirow[t]{3}{*}{$u, v$} & Velocity along $\mathrm{x}, \mathrm{y}$ axes, respectively & $n f$ & Nanofluid \\
\hline & & $f$ & Base fluid \\
\hline & & $s$ & Nano-solid-particles \\
\hline
\end{tabular}

"Corresponding Author Email: houman.babazadehrokni@gmail.com (H. B. Rokni) 


\section{INTRODUCTION}

Internal flow between two plates is one of the most applicable cases in mechanics, civil and environmental engineering. In simple cases, the one-dimensional flow through tube and parallel plates, which is known as Couette-Poisseuille flow, have exact solution, but in general, like most of fluid mechanics make some problems for analytical solution. Nonlinear partial differential equations are known to describe a wide variety of phenomena not only in physics, where applications extend over magneto fluid dynamics, water surface gravity waves, electromagnetic radiation reactions, and ion acoustic waves in plasma, just to name a few, but also in biology and chemistry, and several other fields. It is one of the important tasks in the study of the nonlinear partial differential equations to seek exact and explicit solutions. In the past several decades both mathematicians and physicists have made many attempts in this direction. Various methods for obtaining exact solutions to nonlinear partial differential equations have been proposed. Among these are the B“acklund transformation method, Hirota's bilinear method, the inverse scattering transform method, extended tanh method [1-3], Adomian pade approximation [4-6], Variational method [7-10], The variational iteration method [11-12], Various

Lindstedt-Poincare methods and others [13-15] Many authors have shown interest in studying twodimensional incompressible flow between two inclined plates. Jeffery and Hamel were the first persons who discussed about this problem and so, it is known as Jeffery-Hamel problem, too. After them, many subsequent analysis have been done for this flow discussed in many textbooks $[1,2]$. We know that only a limited number of these problems have precise analytical solution. These nonlinear equations should be solved using other methods. Recently, numerical calculation methods were good means of analyzing the equations related to fluid dynamics and heat transfer, but as the numerical calculation methods were improving, semi-exact analytical methods were greatly developing as well. Most scientists believe that the combination of numerical and semi-exact analytical methods can also end with useful results. Therefore, many different methods have introduced to solve nonlinear equation such as decomposition method [3, 9] $[13,14]$ methods, variational iteration method and our special method RVIM. In 2009 Hesameddini and Latifizadeh [16] proposed a new method based on Laplace transform - Reconstruction of variational iteration method (RVIM) in which the correctional function of the variational iteration method is obtained without using the Variational theory. Therefore in this method the complexity in calculating the Lagrange multiplier has been removed. In this paper, we have applied RVIM to find the approximate solutions of nonlinear differential equations governing the MHD Jeffery-Hamel flow. After introducing the problem of the flow of fluid through a divergent channel by Jeffery [17] and Hamel [18] in 1915 and 1916, respectively, it is called Jeffery-Hamel flow. On the other hand, the term of Magneto hydro dynamic (MHD) was first introduced by Alfvén [19] in 1970. The theory of Magneto hydro dynamics is inducing current in a moving conductive fluid in presence of magnetic field; such induced current results force on ions of the conductive fluid. The theoretical study of (MHD) channel has been a subject of great interest due to its extensive applications in designing cooling systems with liquid metals, MHD generators, accelerators, pumps and flow meters [20-21]. The small disturbance stability of Magneto hydro dynamic stability of planePoiseuille flow has been investigated by Makinde [22] and Motsa [23] for generalized plane Couette flow. Their results show that magnetic field has stabilizing effects on the flow. Considerable efforts have been done to study the MHD theory for technological application of fluid pumping system in which electrical energy forces the working conductive fluid. Damping and controlling of electrically conducting fluid can be achieved by means of an electromagnetic body force (Lorentz force) produced by the interaction of an applied magnetic field and an electric current that usually is externally supplied. Anwari [24] studied the fundamental characteristics of linear Faraday MHD theoretically and numerically, for various loading configurations. Homsy et al. [25] emphasized on the idea that in such problems, the moving ions drag the bulk fluid with themselves and such MHD system induces continues pumping of conductive fluid without any moving part. Taking into account the rising demands of modern technology, including chemical production, power station, and microelectronics, there is a need to develop new types of fluids that will be more effective in nanofluid' is envisioned to describe a fluid in which nanometer-sized particles are suspended is conventional heat transfer basic fluids [26]. At the end a comparison has been provided. The numerical results of this problem are done by using Maple 12. Also this method applied to find the effect of nanoparticle volume fraction on velocity profile.

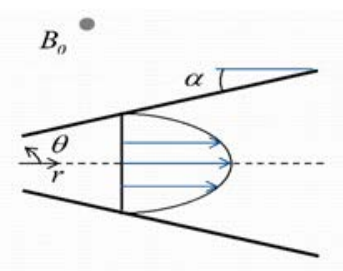

Figure 1. Geometry of the MHD Jeffery-Hamel flow 


\section{GOVERNING EQUATIONS}

Consider a system of cylindrical polar coordinates $(r, \theta, z)$ which steady two-dimensional flow of an incompressible conducting viscous fluid from a source or sink at channel walls lie in planes, and intersect in the axis of $z$. Assuming purely radial motion which means that there is no change in the flow parameter along the $z$ direction. The flow depends on $r$ and $\theta$ and further assume that there is no magnetic field in the z-direction. The reduced form of continuity, Navier-Stokes and Maxwell's equations are [17]:

$\frac{\rho_{n f}}{r} \frac{\partial(r u(r, \theta))}{\partial r}(r u(r, \theta))=0$

$u(r, \theta) \frac{\partial u(r, \theta)}{\partial r}=-\frac{1}{\rho_{n f}} \frac{\partial P}{\partial r}+v_{n f}\left[\frac{\partial^{2} u(r, \theta)}{\partial r^{2}}\right.$

$\left.+\frac{1}{r} \frac{\partial u(r, \theta)}{\partial r}+\frac{1}{r^{2}} \frac{\partial^{2} u(r, \theta)}{\partial \theta^{2}}-\frac{u(r, \theta)}{r^{2}}\right]-\frac{\sigma B_{0}^{2}}{\rho_{n f} r^{2}} u(r, \theta)$

$\frac{1}{\rho_{n f} r} \frac{\partial P}{\partial \theta}-\frac{2 v_{n f}}{r^{2}} \frac{\partial u(r, \theta)}{\partial \theta}=0$

Where $B_{0}$ is the electromagnetic induction, $\sigma$ the conductivity of the fluid, $u(r)$ is the velocity along radial direction, $P$ is the fluid pressure, $v_{n f}$ the coefficient of kinematic viscosity and $\rho_{n f}$ the fluid density. The effective density $\rho_{n f}$, the effective dynamic viscosity $\mu_{n f}$ and kinematic viscosity $v_{n f}$ of the nanofluid are given as [7]:

$\rho_{n f}=\rho_{f}(1-\phi)+\rho_{s} \phi, \quad \mu_{n f}=\frac{\mu_{f}}{(1-\phi)^{2.5}}, \quad v_{n f}=\frac{\mu_{f}}{\rho_{n f}}$

Here, $\phi$ is the solid volume fraction.

$f(\theta)=r u(r)$

Introducing the $\eta=\theta / \alpha$ as the dimensionless degree, the dimensionless form of the velocity parameter can be obtained by dividing that to its maximum value as:

$f(\eta)=\frac{f(\theta)}{f_{\max }}$

Substituting Equation (5) into Equations (2) and (3), and eliminating $\mathrm{P}$, one can obtain the ordinary differential equation for the normalized function profile as [4]:

$f^{\prime \prime \prime}(\eta)+2 \alpha \operatorname{Re} \cdot A^{*}(1-\phi)^{2.5} f(\eta) f^{\prime}(\eta)$

$+\left(4-(1-\phi)^{1.25} H a\right) \alpha^{2} f^{\prime}(\eta)=0$

Where $A^{*}$ is a parameter Reynolds number and Hartmann number based on the electromagnetic parameter are introduced as following form:
$A^{*}=(1-\phi)+\frac{\rho_{s}}{\rho_{f}} \phi$

$\mathrm{Re}=\frac{f_{\max } \alpha}{v_{f}}=\frac{U_{\max } r \alpha}{v_{f}}$

$\left(\begin{array}{l}\text { divergent }- \text { channel }: \alpha>0, f_{\max }>0 \\ \text { convergent - channel }: \alpha<0, f_{\max }<0\end{array}\right)$

$H a=\sqrt{\frac{\sigma B_{0}^{2}}{\rho_{f} v_{f}}}$

With the following reduced form of boundary conditions

$f(0)=1, f^{\prime}(0)=0, f(1)=0$

Physically these boundary conditions mean that maximum values of velocity are observed at centerline $(\eta=0)$ as shown in Figure 1, and we consider fully develop velocity profile, thus rate of velocity is zero at $(\eta=0)$. Also, in fluid dynamics, the no-slip condition for fluid, the no-slip condition for fluid states that at a solid boundary, the fluid will have zero velocity relative to the boundary. The fluid velocity at all fluidsolid boundaries is equal to that of the solid boundary, so the value is zero at $(\eta=1)$.

2. 1. Fundamentals of Reconstruction of Variation Iteration Method (RVIM) In the following section, an alternative method for finding the optimal value of the Lagrange multiplier by the use of the Laplace transform [27] will be investigated a large of problems in science and engineering involve the solution of partial differential equations. Suppose $x, t$ are two independent variables; consider $t$ as the principal variable and $x$ as the secondary variable. If $u(x, t)$ is a function of two variables $x$ and $t$, when the Laplace transform is applied with $t$ as a variable, definition of Laplace transform is:

$L[u(x, t) ; s]=\int_{0}^{\infty} e^{-s t} u(x, t) d t$

We have some preliminary notations as:

$L\left[\frac{\partial u}{\partial t} ; s\right]=\int_{0}^{\infty} e^{-s t} \frac{\partial u}{\partial t} d t=s U(x, s)-u(x, 0)$

$L\left[\frac{\partial^{2} u}{\partial t^{2}} ; s\right]=s^{2} U(x, s)-s u(x, 0)-u_{t}(x, 0)$

$U(x, s)=L[u(x, t) ; s]$

We often come across functions which are not the transform of some known function, but then, they can possibly be as a product of two functions, each of which is the transform of a known function. Thus we may be able to write the given function as $U(x, s), V(x, s)$ 
where $U(s)$ and $V(s)$ are known to the transform of the functions $u(x, t), v(x, t)$ respectively. The convolution of $u(x, t)$ and $v(x, t)$ is written $u(x, t) * v(x, t)$. It is defined as the integral of the product of the two functions after one is reversed and shifted. Convolution Theorem: if $U(x, s), V(x, s)$ are the Laplace transform of $u(x, t), v(x, t)$, when the Laplace transform is applied to $t$ as a variable, respectively; then $U(x, s) . V(x, s)$ is the Laplace Transform of $\int_{0}^{t} u(x, t-\varepsilon) v(x, \varepsilon) d \varepsilon$

$L^{-1}[U(x, s) \cdot V(x, s)]=\int_{0}^{t} u(x, t-\varepsilon) v(x, \varepsilon) d \varepsilon$

To facilitate our discussion of Reconstruction of Variational Iteration Method, introducing the new linear or nonlinear function $h(u(x, t))=f(x, t)-N(u(x, t))$ and considering the new equation, rewrite $h(u(x, t))=$ $f(x, t)-N(u(x, t))$ as :

$L(u(t, x)=h(t, x, u))$

Now, for implementation the correctional function of VIM based on new idea of Laplace transform, applying Laplace Transform to both sides of the above equation so that we introduce artificial initial conditions to zero for main problem, then left hand side of equation after transformation is featured as:

$L[L\{u(x, t)\}]=U(x, s) P(s)$

Where $P(s)$ is polynomial with the degree of the highest order derivative of linear operator

$L[L[u(x, t)]]=U(x, s) P(s)=L[h\{(x, t, u)\}]$

$U(x, s)=\frac{L[h\{(x, t, u)\}]}{P(s)}$

Suppose that $D(s)=1 / P(s) \quad$ and $L[\{h(x, t, u))\}]=H(x, s)$. Using the convolution theorem we have:

$U(x, s)=D(s) \cdot H(x, s)=L\{(d(t) \cdot h(x, t, u))\}$

Taking the inverse Laplace transform on both side of Equation (21),

$u(x, t)=\int_{0}^{t} d(t-\varepsilon) h(x, \varepsilon, u) d \varepsilon$

$u_{0}(x, t)+\int_{0}^{t} d(t-\varepsilon) h(x, \varepsilon, u) d \varepsilon$

And $u_{0}(x, t)$ is initial solution with or without unknown parameters. In absence of unknown parameters, $u_{0}(x, t)$ should satisfy initial boundary conditions.

2. 2. Application First, we consider the initial guesses and auxiliary linear operators in the following form: $f_{0}(\eta)=a \cdot \eta^{2}+1$

Where $a$ is constant, and we will calculate it with considering boundary condition in Equation (11). According to Equation (18) and Equation (7), we have,

$L(f, \eta)=\frac{\partial^{3} f}{\partial \eta^{3}}=h(f, \eta)$

Applying Laplace Transform with respect to independent variable $\eta$ to both sides of Equation (25), setting all the defined artificial initial conditions to zero and using the inverse Laplace Transform, following relation is achieved

$$
\begin{aligned}
& f_{n+1}(\eta)=f_{0}(\eta)+\int_{0}^{s}\left(2 \alpha \operatorname{Re} \cdot A^{*}(1-\phi)^{2.5} f(\eta) f^{\prime}(\eta)\right. \\
& \left.+\left(4-(1-\phi)^{1.25} H a\right) \alpha^{2} f^{\prime}(\eta)\right) \cdot \frac{(\eta-s)^{2}}{2} d s
\end{aligned}
$$

At now we consider Equation (26), apply the boundary conditions to obtain the value of $a$. By substituting this constant parameter in the obtained data, we will have:

$$
\begin{aligned}
f_{1}= & a \eta^{2}+1+1.017916667 \eta^{6} \\
& -0.25\left(2.48980625 a+4.3625 a^{2} \eta^{2}\right) \eta^{4} \\
& +0.4149682708 a \eta^{4}
\end{aligned}
$$

The results of $f_{i}(\eta)$ are shown graphically.

Plotted results are depicted at various $\alpha, \mathrm{Ha}$, and $\mathrm{Re}$ numbers and comparing our results with some earlier works such as ADM illustrated their excellent accuracy of RVIM which introduced as a reliable technique.

\section{RESULTS AND DISCUSSION}

In this study the objective was to apply RVIM to obtain an explicit analytic solution of the MHD Jeffery-Hamel problem. At first viscous fluid $(\phi)$ with magnetic field effect is considered and finally nanofluid flow without magnetic field $(H a=0)$ is investigated. The magnetic field acts as a control parameter such as the flow Reynolds number and the angle of the walls, in MHD JefferyHamel problems. There is an additional nondimensional parameter that determines the solutions, namely the Hartmann number. For comparison, a few limited cases of the ADM solutions compared with the numerical results. The comparison between the numerical results and ADM solution for velocity when $R e=25$ and $\alpha=5^{\circ}$ are shown in Table1.

Figures 2 and 3 show the magnetic field effects on the velocity profiles for divergent channels. There are good agreements between the numerical solution obtained by the fourth-order Runge-Kutta method and ADM. The velocity curves show that the rate of transport is considerably reduced with increase of Hartmann number. 


\begin{tabular}{|c|c|c|c|c|c|c|c|c|c|}
\hline \multirow{2}{*}{$\eta$} & \multicolumn{3}{|c|}{$\mathbf{H a}=\mathbf{0}$} & \multicolumn{3}{|c|}{$H a=250$} & \multicolumn{3}{|c|}{$\mathbf{H a}=500$} \\
\hline & Numerical & ADM & RVIM & Numerical & ADM & RVIM & Numerical & RVIM & Error \\
\hline 0 & 1 & 1 & 1 & 1 & 1 & 1 & 1 & 1 & 1 \\
\hline 0.1 & 0.986671 & 0.986637 & 0.986669331 & 0.988606 & 0.990196 & 0.988605742 & 0.99022 & 0.992695 & 0.990220869 \\
\hline 0.2 & 0.947258 & 0.947127 & 0.947253132 & 0.9547 & 0.960841 & 0.954700449 & 0.960933 & 0.970544 & 0.960936654 \\
\hline 0.3 & 0.883419 & 0.883146 & 0.883409402 & 0.899076 & 0.912079 & 0.899076636 & 0.912273 & 0.912273 & 0.912281699 \\
\hline 0.4 & 0.797697 & 0.797259 & 0.797682307 & 0.822925 & 0.811225 & 0.822926861 & 0.844383 & 0.832683 & 0.844396622 \\
\hline 0.5 & 0.693233 & 0.692638 & 0.693212823 & 0.727664 & 0.713799 & 0.727666691 & 0.757286 & 0.743421 & 0.757305171 \\
\hline 0.6 & 0.573424 & 0.572716 & 0.573401458 & 0.614709 & 0.604866 & 0.614713269 & 0.650719 & 0.643816 & 0.650743237 \\
\hline 0.7 & 0.441593 & 0.44085 & 0.441569310 & 0.485232 & 0.476625 & 0.485236631 & 50 & 0.515303 & 0.523936575 \\
\hline 0.8 & 0.300674 & 0.300013 & 0.3006545894 & 0.33989 & 0.325834 & 0.339895505 & & 0.361234 & 0.375315708 \\
\hline 0.9 & 0.152979 & 0.152552 & 0.152966340 & 0.178555 & 0.17116 & 0.178558841 & 0.202125 & 0.19473 & 0.202142548 \\
\hline 1 & 0 & 0 & 0 & 0 & 0 & 0 & 0 & 0 & 0 \\
\hline
\end{tabular}

This clearly indicates that the transverse magnetic field opposes the transport phenomena. This is due to the fact that variation of $\mathrm{Ha}$ leads to the variation of the Lorentz force due to magnetic field and the Lorentz force produces more resistance to transport phenomena. Under magnetic field the Lorentz force affect in opposite of the momentum's direction that stabilize the velocity profile.

The results show moderate increases in the velocity with increasing Hartmann numbers at small angle $\left(\alpha=2.5^{\circ}\right)$ and difference between velocity profiles are more noticeable at greater angles. Backflow is excluded in converging channels but it may occur for large Reynolds numbers in diverging channels. For specified opening angle, after a critical Reynolds number, we observe that separation and backflow is started (see Figure 2).

Figure 3 shows the magnetic field effects at constant $\alpha$ and different Reynolds numbers. At $\alpha=5^{\circ}, \operatorname{Re}=25$ with increasing Hartmann number the velocity profile becomes flat and thickness of boundary layer decreases, but at this Reynolds number no back flow observed as it is shown in Figure 3(a) .It can be seen in Figure 3(b) that without magnetic field at $\alpha=5^{\circ}, \mathrm{Re}=50$ back flow starts, that with Hartmann number increasing this phenomenon are eliminated. By increasing Reynolds number the back flow expands so greater magnetic field is needed in order to eliminate it. As it is shown in Figures 3(c) and 3(d) $\alpha=5^{\circ}, \operatorname{Re}=75$ that back flow is eliminated at $H a=1000$ while at $\alpha=5^{\circ}, \mathrm{Re}=100$ this occurs in $\mathrm{Ha}=2000$.
Finally we consider a $\mathrm{Cu}$-Water nano fluid flow and investigate effect of nanoparticle volume fraction. It is assumed that the base fluid and the nanoparticles are in thermal equilibrium and no slip occurs between them. The density of water is $\rho_{f}=997.1$ and density of $\mathrm{Cu}$ is $\rho_{s}=8933$. It can be seen from Figure 4 that increasing in nanoparticle volume fraction leads to increase in momentum boundary layer thickness as a result of an increase in the energy transportation the fluid with the increasing of volume fraction. Also it can be seen that increasing nanoparticle volume fraction as high values of Reynolds number and angle of the channel leads to start back flow (see Figure 4).

\section{CONCLUSION}

In this present work RVIM successfully applied to obtain an explicit analytic solution of the magneto hydrodynamic Jeffery-Hamel flow, also this problem is solved by a numerical method (the Runge-Kutta method of 4th order), and effect of adding nanoparticle in the absence of magnetic field is considered. in continue, comparison results via exact solution and the ADM's obtained results illustrated to us capability of RVIM As we can see the RVIM has high accuracy and simple for nonlinear problems like Jeffrey - Hamel flow .and finally present some graphs to depict at various $\alpha$, Ha, and Re numbers that more confirm this claim. 


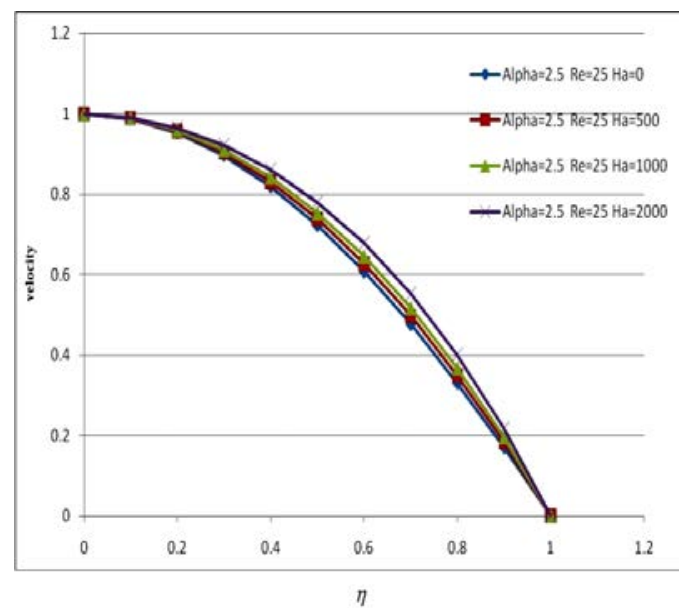

2 (a)

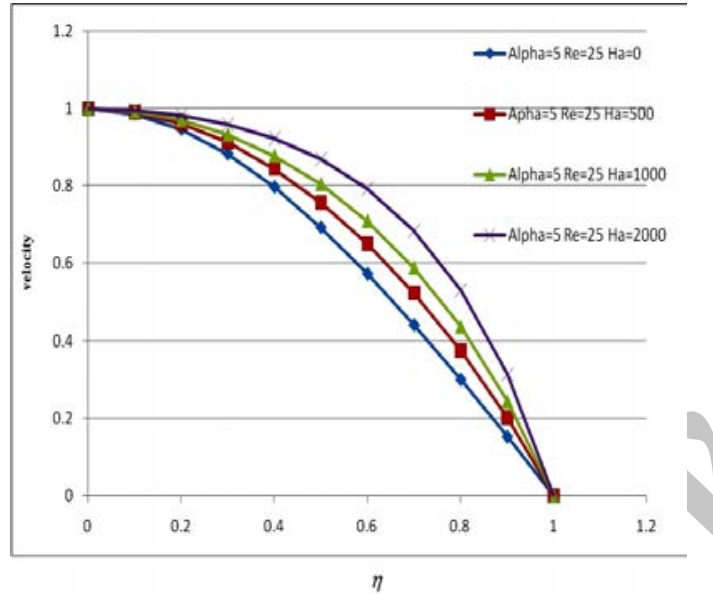

2 (b)

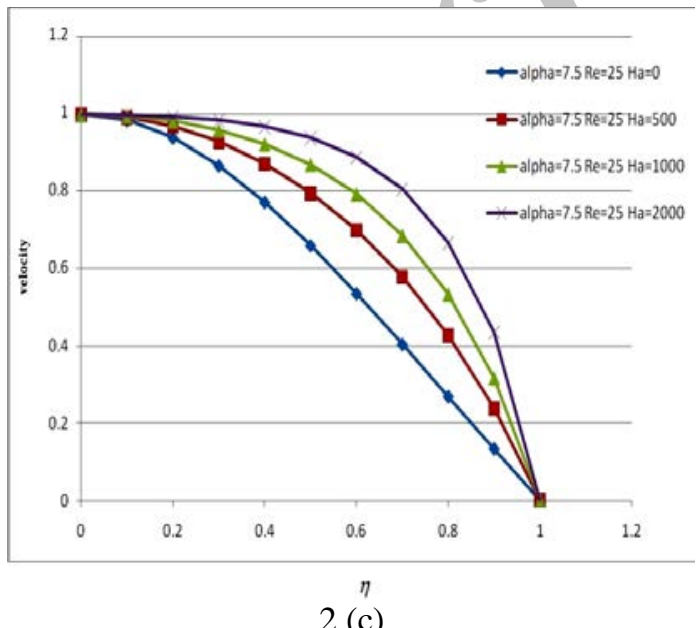

2 (c)

Figure 2. The ADM solution for velocity in divergent channel for (a) $\alpha=2.5^{\circ}, \operatorname{Re}=25$ and $\phi=0$, (b) $\alpha=5^{\circ}, \operatorname{Re}=25$ and $\phi=0$ and (c) $\alpha=7.5^{\circ}, \operatorname{Re}=25$ and $\phi=0$.

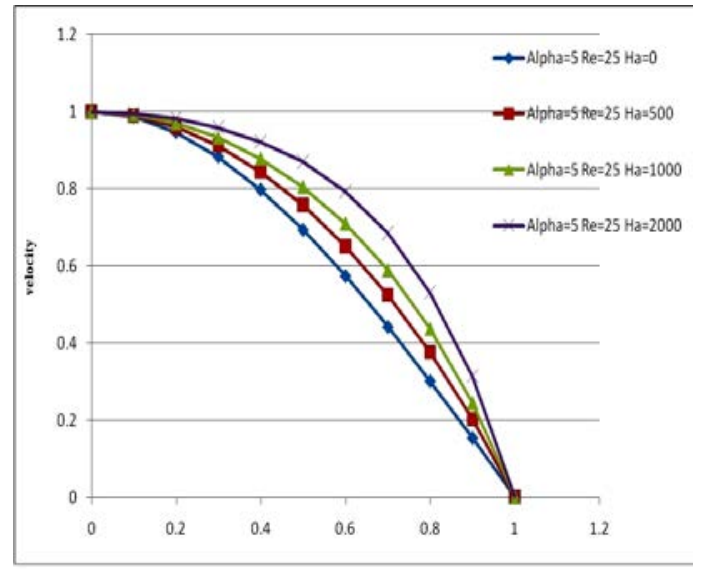

$3($ a)

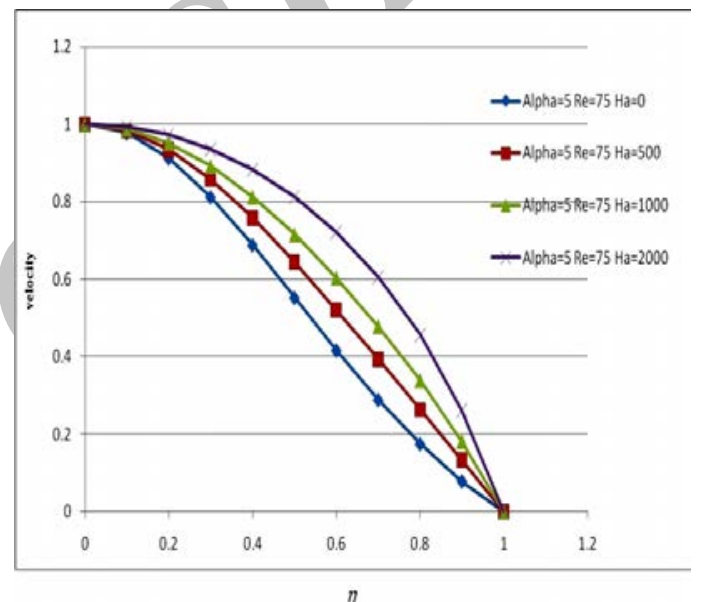

3 (b)

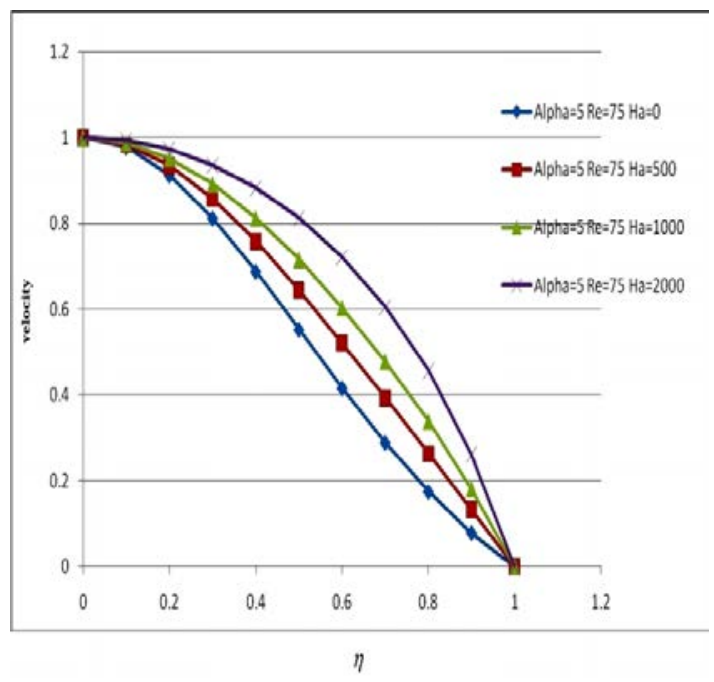

$3(\mathrm{c})$ 


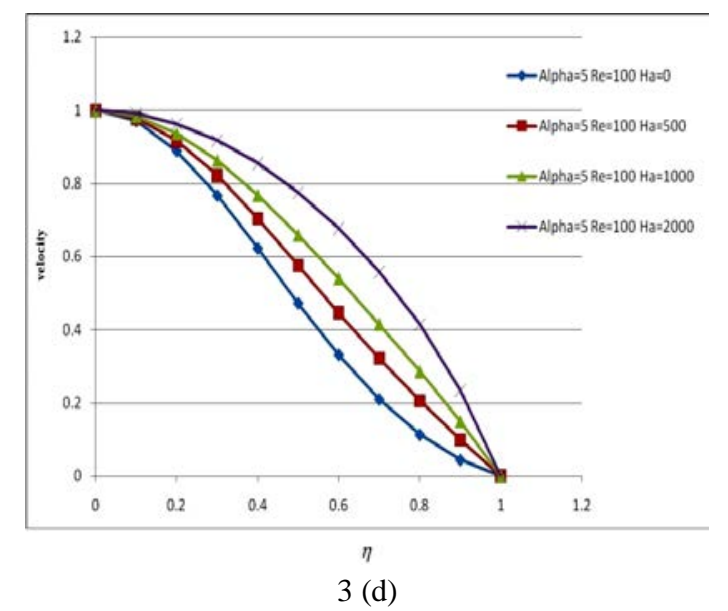

Figure 3. The ADM solution for velocity in divergent channel for (a) $\alpha=5^{\circ}, \operatorname{Re}=25$ and $\phi=0$, (b) $\alpha=5^{\circ}, \operatorname{Re}=50$ and $\phi=0$, (c) $\alpha=5^{\circ}, \operatorname{Re}=75$ and $\phi=0$ and (d) $\alpha=5^{\circ}, \operatorname{Re}=100$ and $\phi=0$.

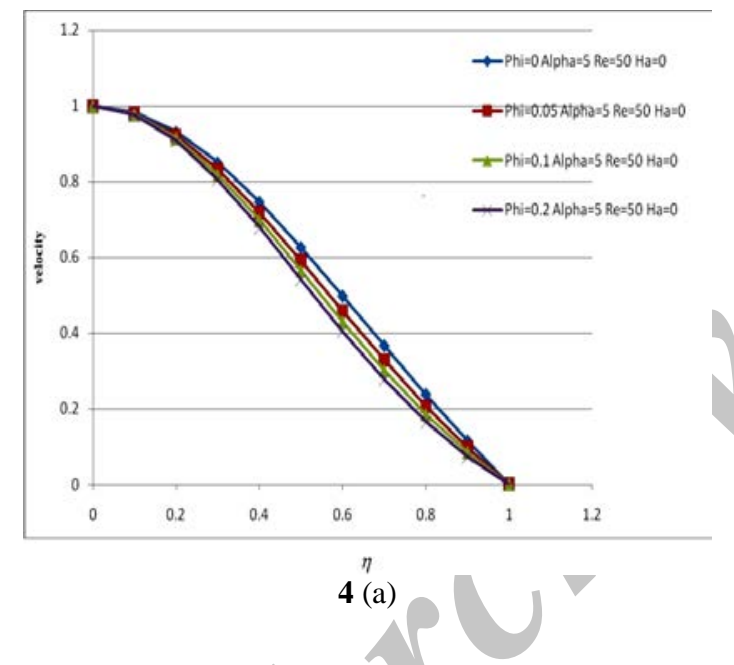

\section{REFERENCES}

1. Schlichting, H., Boundary-Layer Theory. McGraw-Hill, New York, 2000

2. White, F.M., Viscous Fluid Flow, McGraw-Hill, 1991.

3. Domairry, G. , Mohsenzadeh, A. and Famouri, M., "The application of homotopy analysis method to solve nonlinear differential equation governing Jeffery-Hamel flow", Communications in Nonlinear Science and Numerical Simulation, Vol. 14( 1), (2009), 85-95

4. Sadighi. A., Ganji, D. D." Analytic treatment of linear and nonlinear Schrödinger equations: A study with homotopyperturbation and Adomian decomposition methods" Physics Letters A, Vol. 327 (2008) 465-469.

5. Hosein Nia, A. H, Soltani, H. , Ghasemi, J., Rabjbar, A. N. and Ganji, D.D. , "Maintaining the stability of nonlinear differential equations by the enhancement of HPM", Physics Letters A, Vol. 372( 16), (2008), 2855-2861.

6. Esmaelipour, M. and Ganji, D. D. , “Application of He's homotopy perturbation method to boundary layer flow and
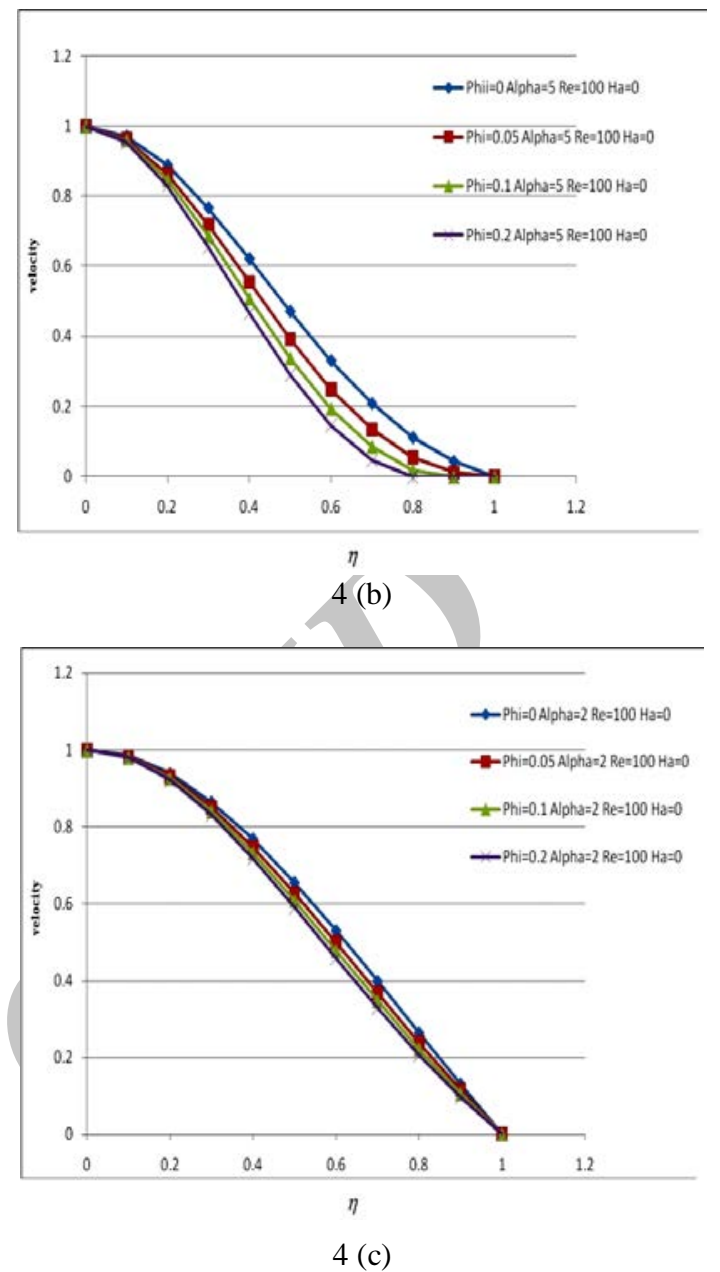

Figure 4. Effect of nanoparticle volume fraction on velocity profiles for $\mathrm{Cu}$-Water.

convection heat transfer over a flat plate", Physics Letters A, Vol. 372(1), (2007), 33-38

7. Ganji, D. D., Afrouzi, G. A., Hosseinzaseh, H. , Talarposhti, R. A. , "Approximation of analytical solution", Physics Letters A, Vol. 371 (2007) 20. 2615.

8. Ganji, D. D., Tari, H. and Bakhshi Jooybari, M. , "Variational iteration method and homotopy perturbation method for nonlinear evolution equations" Computers \& Mathematics with Applications, Vol. 54 (7-8), (2007) 1018-1027.

9. Ganji, D. D., Nourollahi, M. and Mohseni, E. "Application of He's methods to nonlinear chemistry problems", Computers \& Mathematics with Applications, Vol. 54 (7-8), (2007) 11221132

10. Ganji, D.D., Hosseini, M. J., Shayegh, J. ,"Some nonlinear heat transfer equations solved by three approximate methods", International Communications in Heat and Mass Transfer, Vol. 34 (8), (2007), 1003-1016.

11. Ganji, D. D. and Sadighi, A., "Application of homotopyperturbation and variational iteration methods to nonlinear heat transfer and porous media equations", Journal of Computational and Applied Mathematics, Vol. 207(1), (2007), 24-34 
12. Ganji, D.D., "The application of He's homotopy perturbation method to nonlinear equations arising in heat transfer", Physics Letters A, Vol. 355 (4-5), (2006), 337-341.

13. Liao, S. J., Ph.D thesis, Shanghai Jiao Tong University, 1992.

14. Fakhari, A. , Domairry, G. , Ebrahimpour, "Approximate explicit solutions of nonlinear BBMB equations by homotopy analysis method and comparison with the exact solution" Physics Letters A, Vol.368 (1-2) (2007) 64-68.

15. Adomian, G. "'A review of the decomposition method in applied mathematics", Journal of Mathematical Analysis and Applications, Vol. 135 (2), (1988), 501-567

16. Hesameddini, E., Latifizadeh, H., "Reconstruction of variational iteration algorithms using the Laplace transform," International. Journal of Nonlinear Science, Vol. 10, (2009), 1377-1382.

17. Jeffery, G. B., "The two-dimensional steady motion of a viscous fluid", Philosophical Magazine. Vol. 6, (1915), 455-465

18. Hamel, G., "Spiralförmige Bewgungen Zäher Flüssigkeiten, Jahresber Deutsch", Mathematical Verein. Vol. 25, (1916), 3460.

19. Bansal, L., "Magnetofluiddynamics of Viscous Fluids Jaipur Publishing House", Jaipur,India, OCLC 70267818, (1994).

20. Cha, J. E., Ahn,Y. C., Moo-Hwan Kim, "Flow measurement with an electromagnetic flowmeter in two-phase bubbly and slug flow regimes", Flow Measurement and Instrumentation, Vol. 12 (5-6), (2002), 329-339.

21. Tendler, M., "Confinement and related transport in extrap geometry", Nuclear Instruments and Methods in Physics Research, Vol. 207 (1-2), (1983), 233-240.
22. Makinde, O. D., Motsa, S. S., "Hydromagnetic stability of plane Poiseuille flow using Chebyshev spectral collocation method", International Journal of Mathematics and Computer Science, Vol. 12 (2), (2001), 175-183,

23. Makinde, O. D., "Magneto-hydrodynamic stability of planePoiseuille flow using multi-deck asymptotic technique", Mathematical and Computer Modelling, Vol. 37 (3-4), (2003), 251-259,

24. Anwari, M., Harada, N., Takahashi, S., "Performance of a magnetohydrodynamic accelerator using air-plasma as working gas", Energy Conversion Management, Vol. 4, (2005), 26052613.

25. Homsy, A., Koster, S., Eijkel, J. C. T., Ven der Berg, A., Lucklum, F. , Verpoorte, E., de Rooij, N. F., "A high current density DC magnetohydrodynamic (MHD) micropump", $\boldsymbol{L} \boldsymbol{a} \boldsymbol{b}$ on a Chip. Royal Society of Chemistry. Vol. 5, (2005), 466-471,

26. Kakaç, S., Pramuanjaroenkij, A., "Review of convective heat transfer enhancement with nanofluids", International Journal of Heat and Mass Transfer. Vol.52 (13-14), (2009), 31873196 ,

27. Imani, A. A., Ganji, D. D., Rokni, H. B., Latifizadeh, H., Hesameddini, E., Hadi Rafiee, M., "Approximate traveling wave solution for shallow water wave equation", Applied Math Modeling, Vol. 36, (2012), 1550-1557.

\title{
Analytical Investigation of Jeffery-Hamel Flow with High Magnetic Field and Nano Particle by RVIM
}

\author{
A. A. Imani a , Y. Rostamian b, D. D. Ganji c, H. B. Rokni d \\ ${ }^{a}$ Islamic Azad University, Babol Branch, Babol, Iran \\ ${ }^{b}$ Faculty of Mechanical Engineering, Islamic Azad University, Sari Branch, Sari, Iran \\ c Department of Mechanical Engineering, Babol University of Technology, P.O. Box 484, Babol, Iran. \\ ${ }^{d}$ University of Denver, Mechanical and Materials Engineering, 2390 S York St, Denver, CO, USA.
}

\section{$P A P E R \quad I N F O$}

\section{Paper history:}

Received 23 June 2012

Received in revised form 25 August 2012

Accepted in revised form 30 August 2012

\section{Keywords:}

Magneto Hydro Dynamic

Jeffery-hamel Flow

RVIM

Nonlinear Ordinary Differential Equation Nanofluid

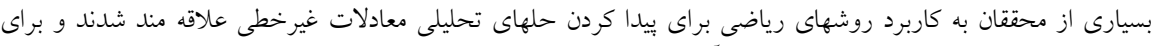

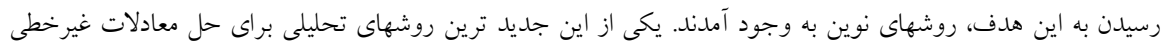

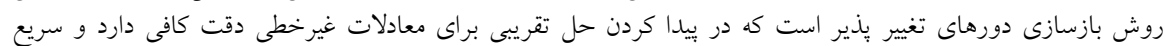

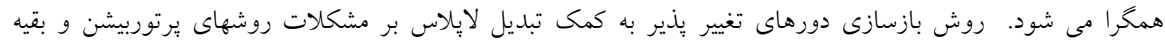

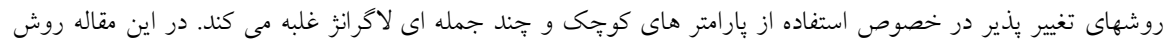

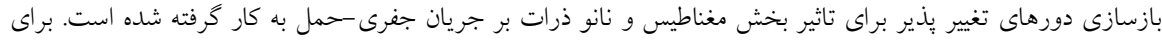

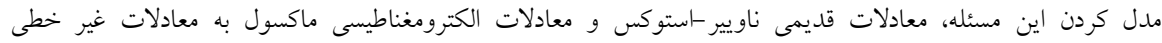

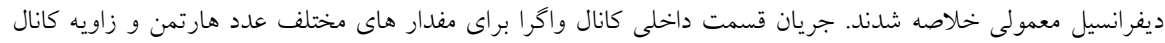

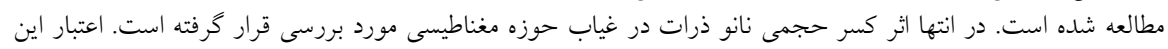

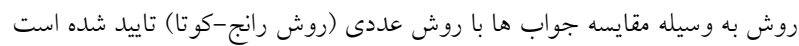

doi: $10.5829 /$ idosi.ije.2012.25.03c.09 


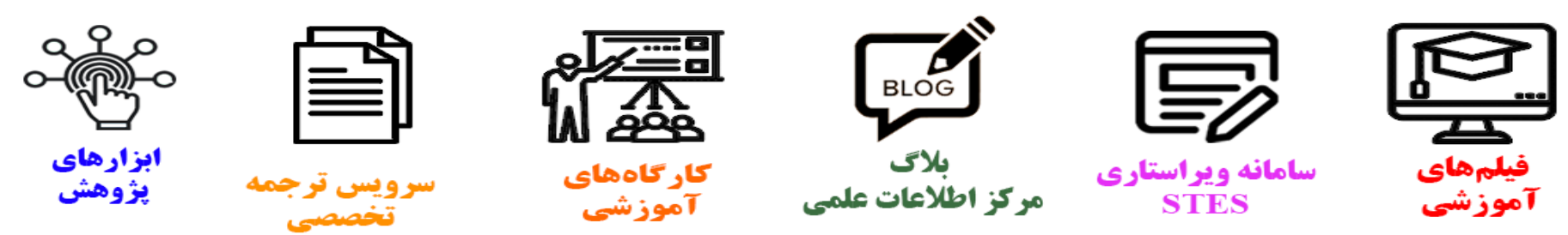

\section{(c)}

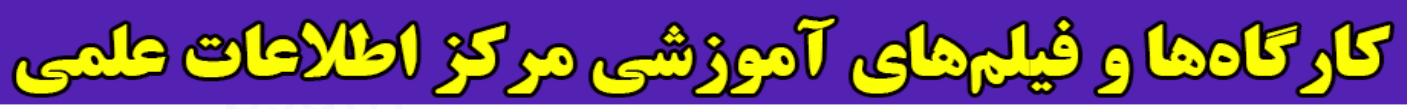
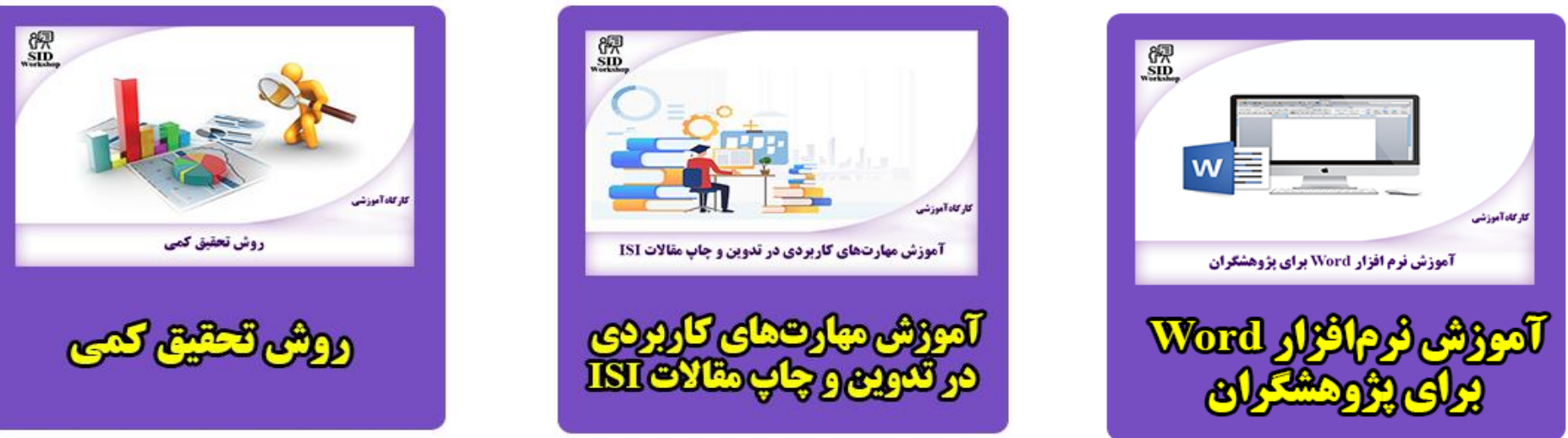